\title{
Application of MGC method to centering saturated and stretched images $\dagger$
}

\author{
Y. D. Mao ${ }^{1,2}, \mathbf{Y} \cdot \mathbf{L i}^{1,2}$ and Z. H. Tang ${ }^{1}$ \\ ${ }^{1}$ Shanghai Astronomical Observatory, Chinese Academy of Sciences, Shanghai 200030, China \\ email: dundun@shao.ac.cn \\ ${ }^{2}$ Graduate School of the Chinese Academy of Sciences, Beijing 100039, China
}

\begin{abstract}
Sometimes saturated and stretched star images with bad data in their central parts may result in astronomical CCD observations. Considering the special characteristics of the saturated and stretched star images, a method known as MGC(Mean-Geometric-Centering) is put forward for determining the centers of these images. The basic principles and processing pipelines of MGC are introduced, and several noteworthy items in the realization of MGC are discussed. Results of applying MGC to simulated and real data are also presented.
\end{abstract}

Keywords. techniques: image processing, methods: data analysis, astrometry

\section{Introduction}

Due to the restriction of linear response range, long-time exposure usually leads to saturated images, especially when using photographic plates we did in the early days. Because artificial satellites and stars have different motions, stretched images always exist whether we are tracking satellites or stars. None of the existing methods(including modified moment, Gaussian function fit, median, and derivative search, etc. Stone(1989)) is available for determining the centers of the saturated and stretched images. Based on the principle of marginal extraction from images smeared with noise, we come up with a new method known as MGC for centering these images. According to the results of applying MGC to simulated as well as real data obtained with $1.56 \mathrm{~m}$ telescope at Shanghai, MGC was found to be better than the other methods for centering the images.

\section{Pipelines and processing results of MGC}

The basic principles and pipelines of MGC are as follows:

- Extract a series of edges, starting from the outside and moving to the inside of the saturated or stretched images with growing thresholds that are increased step by step.

- Link the extracted edges and get a series of closed curves that are just like contours of the images, corresponding to different thresholds.

- Calculate the geometric centers of these closed curves. According to an analysis of empirical data, when SNR is greater than certain threshold SNR0, the geometric centers $(\mathrm{xc} 1, \mathrm{yc} 1),(\mathrm{xc} 2, \mathrm{yc} 2),(\mathrm{xc} 3, \mathrm{yc} 3), \ldots(\mathrm{xcn}, \mathrm{ycn})$ are found to be very stable for thresholds in some particular range [TL, TH].

- Average the geometric centers with thresholds in the range [TL,TH] to get new coordinates $(\mathrm{xc}, \mathrm{yc})$, which we take as the center coordinate of the saturated or stretched star images.

$\dagger$ Supported by the National Natural Science Foundation of China (Grant Nos. 10673026, 10333050, and 10573018) and Science Technology Commission of Shanghai Municipality(06DZ22101). 
Table 1 Results with for centering method for saturated star images

\begin{tabular}{ccccc}
\hline Methods & $\sigma_{x}$ & $\sigma_{y}$ & $\delta_{x}$ & $\delta_{y}$ \\
\hline Moment & & & 1.8017 & 1.3749 \\
2D Gaussian & 0.1058 & 0.1082 & 0.6396 & -0.1294 \\
1D Gaussian & 0.3153 & 0.1439 & -0.3323 & -0.3926 \\
MGC & 0.1294 & 0.0619 & -0.1017 & -0.1500 \\
\hline
\end{tabular}

Table 2 Results with two centering method for stretched images

\begin{tabular}{ccc}
\hline FITS & Moment & MGC \\
\hline $1013 \mathrm{i} 13$ & $0.98713 \pm 0.00368$ & $0.99231 \pm 0.00079$ \\
& $-0.12031 \pm 0.00368$ & $-0.12018 \pm 0.00079$ \\
& $0.17384 \pm 0.04564$ & $0.26934 \pm 0.01667$ \\
& $-0.04317 \pm 0.04564$ & $0.02710 \pm 0.01667$ \\
\hline Nref=4 & $\sigma_{\alpha} \cos \delta=1.220$ & $\sigma_{\alpha} \cos \delta=0.262$ \\
& $\sigma_{\delta}=0.512$ & $\sigma_{\delta}=0.187$ \\
\hline $0925 \mathrm{i} 100$ & $0.98732 \pm 0.00301$ & $0.99350 \pm 0.00040$ \\
& $0.12031 \pm 0.00301$ & $-0.12024 \pm 0.00040$ \\
& $0.02380 \pm 0.05775$ & $0.02586 \pm 0.00526$ \\
Nref $=4$ & $0.11815 \pm 0.05775$ & $-0.01775 \pm 0.00526$ \\
& $\sigma_{\alpha} \cos \delta=1.292$ & $\sigma_{\alpha} \cos \delta=0.174$ \\
\hline
\end{tabular}

In comparison with other centering methods, we applied MGC to centering a large number of saturated and stretched images. Table 1 and Table 2 contrast the results obtained with different methods for the saturated and stretched star images respectively. According to our experience, a linear model with four parameters is usually good enough. Tycho-2 is used as a reference star catalogue (Høg 1999).

\section{Conclusion}

- The SNR of star images is a major factor for the centering accuracy. MGC is found to be much better than the other conventional algorithms for centering saturated and, especially, stretched star images.

- Usually star images obtained through marginal extraction are seriously infected with imperfections due to cosmic rays, hot pixels and bad pixels, etc., which may directly affect the processing results of MGC. Hence it is very important to preprocess the images with an appropriate filtering method. To avoid distorting the original images, we suggest using a median filter.

- Although MGC has many advantages as mentioned above for centering the stretched images, there are still some shortcomings. For example, too many man-induced factors have been included in determining SNR0 and [TL,TH]. In order to be more objective, we need to first experiment with simulated data that were generated with identical observing conditions, and then determine the SNR0 and [TL,TH] values through an analysis of the experiment results.

\section{References}

Stone R. C., et al. 1989, AJ, 97, 1227

$\mathrm{H} \varnothing \mathrm{g}$ E., et al. 1999, A\&GA, 357, 367 